
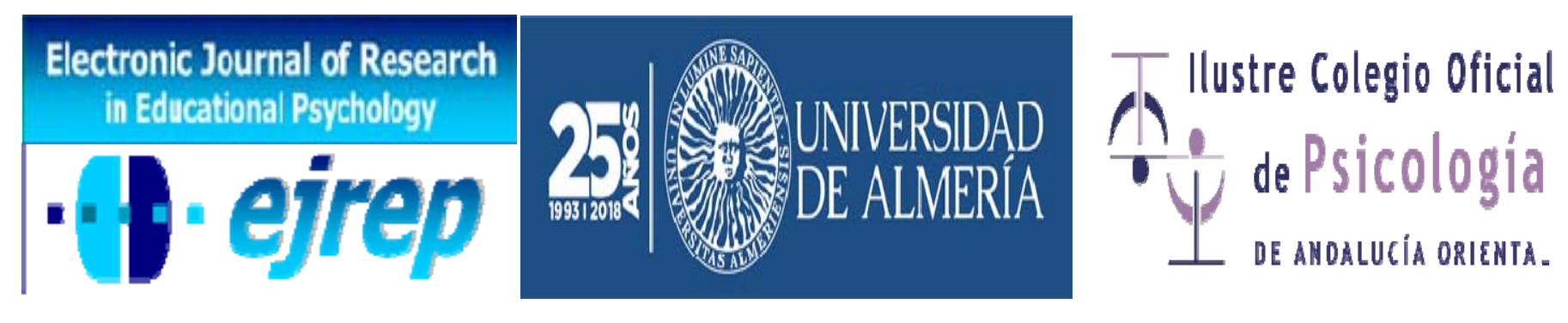

\title{
Mejora de las competencias socioemocionales en el aula, con jóvenes de Educación Secunda- ria, mediante el programa SEA
}

\section{César Rodríguez-Ledo ${ }^{1}$, Santos Orejudo Hernández ${ }^{2}$, Laura Celma Pastor ${ }^{3}, \mathrm{M}^{\mathrm{a}}$ Jesus Cardoso Moreno ${ }^{2}$.}

${ }^{1}$ Departamento Ciencias de la Educación. Universidad de Zaragoza, Zaragoza

${ }^{2}$ Departamento Psicología y Sociología. Universidad de Zaragoza, Zaragoza

${ }^{3}$ Neuroactive. neurotecnología para la mejora cognitiva, Zaragoza

\section{España}

Correspondencia: César Rodríguez Ledo. C/ Pedro Cerbuna 12, 50009 Zaragoza, España. E-mail: cesarrledo@gmail.com

\footnotetext{
(C) Universidad de Almería and Ilustre Colegio Oficial de la Psicología de Andalucía Oriental (Spain)
} 


\section{Resumen}

Introducción. En este trabajo se presenta la evaluación del impacto de un programa de desarrollo de las competencias emo-cionales en la base del modelo teórico SEA (atención y comprensión emocional, regulación y reparación emocional y expresión social adaptativa de las emociones) sobre tales competencias en jóvenes adolescentes y pre-adolescentes.

Método. El programa se implementó en un IES público durante un curso escolar por parte de los propios profesores-tutores de los alumnos. Los participantes de este estudio son 156 alumnos de 11 a 14 años estudiantes de $1^{\circ}$ de E.S.O. en sus di-ferentes condiciones experimentales y control. Se ha utilizado un diseño cuasiexperimental pretest-postest con grupo control para evaluar la eficacia del programa. Las pruebas usadas para ello miden las competencias socioemocionales, la empatía y el ajuste social de los participantes. Además, se hace una medida de seguimiento 3 meses después de la finalización de la implementación del programa.

Resultados. La investigación demuestra una mejora significativa en las competencias socioemocionales de los participantes del programa, especialmente en las competencias más relacionadas con la expresión social adaptativa de las emociones. Además, se descubren mejoras también significativas en empatía y ajuste social.

Discusión y conclusiones. Se corrobora la posibilidad de potenciar las competencias socioemocionales mediante la aplicación de un programa de intervención directa en el aula durante un curso escolar y el programa SEA se convierte en candidato para alcanzar este objetivo.

Palabras Clave: Educación socioemocional, empatía, ajuste social, evaluación de programas, adolescentes. 


\begin{abstract}
Introduction. This paper shows the impact evaluation of a social and emotional competences development programme based on the theoretical model SEA (attention and emotional understanding, regulation and emotional repair and adaptive social expression of emotions) on such young adolescent and pre-adolescent competences.

Method. The programme was implemented in a public high-school during a school year the teachers-tutors of the students. The participants of this study are 156 youngsters aged from 11 to 14 years old, all of them students of the first year of the Spanish secondary education system. The design used to evaluate the effectiveness of the programme was quasi-experimental with pre/post-test measurements and with a control group. The tests used for the evaluation assessed variables such as socio-emotional competencies, empathy and the social adjustment of the participants. In addition, a follow-up assessment was carried out 3 months after finalization of programme implementation.
\end{abstract}

Results. The research demonstrates a significant improvement in the socio-emotional competences of program participants, especially in the competencies most related to the adaptive social expression of emotions. There are also significant improvements in empathy and social adjustment.

Discussion and Conclusion. The possibility of strengthening socio-emotional competencies through the application of a direct intervention pro-gram in the classroom during a school year is confirmed and the SEA programme becomes a candidate to achieve this goal.

Keywords: Social-emotional education, empathy, social adjustment, programme evaluation, adolescents. 


\section{Introducción}

El desarrollo de competencias socioemocionales se ha convertido en los últimos años como un objetivo clave a alcanzar para muchos educadores y expertos en desarrollo personal. La potenciación de este grupo de habilidades, justificado en las necesidades actuales de nuestra sociedad (Bisquerra, 2003), se presenta no ya sólo como una herramienta útil, sino como una básica para garantizar un adecuado ajuste social de los alumnos a los centros educativos (Rodríguez-Ledo y Ruíz-Aranda, 2017). Afortunadamente cada vez son más los formadores que así lo creen y trabajan de manera activa el desarrollo de la inteligencia emocional (IE) o socioemocional (ISE) de sus alumnos (CASEL, 2015). Este incremento de acciones educativas, investigaciones, publicaciones y congresos en relación a esta temática aporta cada vez más luz sobre el campo, tanto desde la teoría como desde la aplicación práctica de programas.

Inteligencia socioemocional, ajuste social y mejoras asociadas a su potenciación.

Para empezar a trabajar en potenciar el desarrollo de la ISE es importante operativizar su comprensión. Si entendemos la emoción como un evento interno que energiza nuestra conducta de modo que respondemos al medio acercándonos o alejándonos en función de si las emociones tienen un tono hedónico positivo y negativo (Ekman y Davidson, 1994) y la inteligencia como la capacidad de adaptación al medio, la inteligencia socioemocional quedaría definida como la capacidad de las personas para percibir, comprender, regular y expresar tales eventos emocionales de un modo adaptativo. Por lo tanto, la ISE es un constructo que explica como las emociones son percibidas, reguladas y expresadas con mayor o menor pericia.

Este constructo se ha relacionado con muchas variables, pero hay un área especialmente determinante para contextos educativos y donde la ISE tiene un fuerte poder explicativo: el ajuste social al centro y el bienestar personal consecuencia del mismo. Empezando por el final, se conoce la influencia de la ISE en el bienestar psicológico de las personas (Zeidner y Matthews, 2016). Específicamente, se ha argumentado que los alumnos que puntúan más alto en las medidas de ISE podrían enfrentarse mejor a los estresores y demandas diarias del centro educativo y serían, por tanto, sujetos que informarían de un mayor bienestar percibido que otros con menor desarrollo socioemocional (Bar-On, 2006; Akerjordet y Severinsson, 2007). En la adolescencia, la literatura pone de manifiesto que aquellos con mejores habilidades socioemocionales muestran un mejor ajuste emocional, menos problemas internalizados, 
menos ansiedad y depresión, así como mejores estrategias de afrontamiento (Gomez-Baya, Mendoza, Paíno y Gaspar de Matos, 2017; Resurrección, Salguero y Ruiz-Aranda, 2014). También Martín, Harillo y Mora (2008) encuentran resultados que sugieren una relación directa entre IE percibida e indicadores de bienestar tales como satisfacción vital y felicidad, además de encontrarse una relación inversa entre IE percibida y depresión o ansiedad estado.

Además del bienestar personal, se ha relacionado en diversos estudios la ISE con el ajuste a social inmediato de los jóvenes (Fernández-Berrocal y Ruiz-Aranda, 2008). Ciarrochi, Chan y Bajgar (2001) indican que los adolescentes con alta ISE eran más capaces de establecer y mantener relaciones interpersonales y más satisfactorias, mientras que Lopes, Salovey y Straus (2003) indican que aquellos jóvenes que manejan mejor sus emociones muestran mayor número de interacciones sociales y más positivas. Por otro lado, Schutte, Malouff, Bobik et al. (2001) observan como los alumnos con bajos niveles de ISE suelen presentar mayores niveles de impulsividad y peores habilidades interpersonales, lo cual favorece el desarrollo de diversos comportamientos antisociales que provocan un menor ajuste social. Es lógico pensar que una persona con buenas competencias de ISE pueda reconocer las emociones de los demás con cierta precisión y, probablemente, sepa actuar en consecuencia, ofreciendo información emocional (expresión emocional) adecuada y adaptada al contexto social en el que se encuentra. Precisamente el reconocimiento de las emociones ajenas es algo que se ha estudiado con gran interés en términos de empatía. Se sabe que esta competencia personal incluye tanto respuestas emocionales como experiencias vicarias o, lo que es lo mismo, capacidad para diferenciar entre los estados afectivos de los demás y la habilidad para tomar una perspectiva tanto cognitiva como afectiva respecto a los demás (Feshbach, 1978). La fuerte relación de empatía e ISE hace que en ocasiones se incluyan modelos de ISE como una dimensión más (Goleman, 1998), mientras que en otros modelos se analiza como un componente socioemocional específico incluido en otra dimensión general (Mayer et al., 2000; Bar-On, 2006) o como un rasgo específico (Petrides y Furnham, 2000). Sea como fuere considerada, la empatía se ha relacionada consistentemente no solo con ISE, sino también con aspectos de ajuste social como la estimulación de la amistad y a cuanto ayuda a mantener amistades, a la mejora en la calidad de las relaciones familiares, a la promoción de la salud mental, al comportamiento prosocial, a la colaboración y al altruismo (Sanz de Acebo et al., 2003).

Si la ISE está asociada a aspectos claves del desarrollo personal como la habilidad empática además de otros relacionados con el ajuste social que determina en parte el bienestar 
del alumno de un centro, sería lógico pensar que existan intervenciones que encuentren mejoras tales cuando se trabaja la ISE con alumnos, es decir que potencian su educación socioemocional (ESE a partir de ahora). En efecto a la luz de ESE, han surgido programas e intervenciones varias que buscan desarrollar las competencias socioemocionales de las personas con las que trabajan en base a diferentes modelos de ISE. Estos diferentes programas e intervenciones tienen características diferentes tanto de forma como de fondo, y pese a ello en muchos casos obtienen resultados muy positivos. En un interesante meta-análisis sobre 213 programas que de ESE para alumnos entre 5 y 18 años en el marco de sus centros educativos, Durlak, Weissberg, Dymnicki, Taylor y Schellinger (2011) descubren mejoras significativas en su nivel de ISE general demás de en actitudes más adaptativas, comportamiento social positivo, reducción de problemas de conducta, menor angustia emocional y mayor rendimiento académico. También Greenberg et al. (2003) realizan un meta-análisis, descubriendo como este tipo de programas sirven tanto como intervenciones de mejora, como a modo de factores protectores que disminuyen los problemas de conducta y como bases para un desarrollo saludable. Este efecto protector de los programas de ESE se ha relacionado con como mediante intervenciones que buscan potenciar la ISE se consigue reducir el impacto de problemas de aparición típica en la adolescencia como problemas conductuales, de relación, de uso de sustancias, de comportamientos antisociales o incluso de descenso de la autoestima (Mariscal, Giménez-Dasí, Carriedo y Corral, 2009; Pasch et al., 2008). En este sentido, se ha indicado que mediante el entrenamiento en ISE se pueden prevenir la aparición de conductas violentas, impulsividad y desajuste emocional (Extremera y Fernández-Berrocal, 2003) e incluso futuros problemas de salud mental (Humphrey et al., 2007).

El ajuste psicosocial y la salud mental de los alumnos también se han relacionado con desarrollo socioemocional mediante programas de intervención en el aula. Durlak y Wells (1997) analizan 177 programas de prevención primaria de problemas de comportamiento y sociales en jóvenes menores de 18 años, encontrando mejora en competencias tales como asertividad, habilidades comunicativas y auto-confianza, además de menores problemas internalizantes (ansiedad, depresión, somatización, etc.) y externalizantes (hiperactividad, agresión, problemas de conducta, etc.). En el mismo sentido, Wilson y Lipsey (2007) reportan mejoras en problemas internalizantes en jóvenes tras el trabajo en estas competencias. En concreto el meta-análisis realizado sobre 249 programas de ESE en todas las etapas educativas descubre que estos programas encuentran resultados en forma de efectos en variables relacionadas con el comportamiento social tales como agresión, comportamiento antisocial y 
habilidades sociales, además de tener un mejor ajuste psicosocial al centro educativo. También Durlak, Weissberg, y Pachan (2010), descubren mediante un nuevo meta-análisis como los programas de ESE mejoran de manera significativa indicadores de ajuste conductual tales como disminución de problemas de comportamiento, incremento de comportamientos sociales positivos y un menor uso de drogas.

\section{Buenas prácticas en potenciación de la ISE}

Con el objetivo de diseñar e implementar un programa de potenciación de la ISE efectivo, lo primero que se ha de tener en cuenta es la existencia de una fase previa en la que se logre el compromiso de todos los participantes y conseguir un plan conjunto de acción (Brackett et al., 2009). Tras esto se formará a los aplicadores en el modelo teórico en el que se basa el programa y en las técnicas concretas en las que se basa su implementación. Debemos asegurarnos de que el programa elegido, o diseñado, cumple con lo que Durlak et al. (2011) denominan S.A.F.E. (sequenced, active, focused and explicit). Esto significa que se trata de un programa secuenciado (sequenced), es decir que aplica un conjunto planificado de actividades para desarrollar habilidades de forma secuencial, de forma que unas habilidades se construyan como base de las siguientes. También ha de ser activos (active), o sea que use formas activas de aprendizaje, como los juegos de rol o ensayo conductuales con retroalimentación donde los alumnos son los principales actores de las actividades, que han de ser eminentemente prácticas. Por otro lado, han de ser focalizados (focused), o sea que el programa esté organizado en torno a actividades que presentan unos objetivos concretos a desarrollar, objetivos a los que se dedica el suficiente tiempo para su desarrollo. De igual modo, el programa en su conjunto dedica tiempo suficiente para el desarrollo de las competencias socioemocionales objetivo como, por ejemplo, durante uno o dos cursos escolares. Por último, serán explícitos (explicit) o dirigidos hacia del desarrollo de habilidades sociales y emocionales específicos, de modo que se potencia la ISE en base a un modelo teórico claro donde, además, los alumnos son conscientes en todo momento de que competencia socioemocional están trabajando. Los programas que cumplen con S.A.F.E. son los que, en cada ocasión, reportan mejores resultados que los que no lo cumplen (Durlak et al., 2011). Así, programas que cumplen con un orden concreto de aplicación que usan estrategias de aprendizaje activo y constructivo, que se centran en torno a un número finito y claro de competencias y que potencian el esfuerzo consciente y explícito de los alumnos cuya ISE deseamos que mejore son los que, potencialmente, pueden obtener unos resultados interesantes y significativos en comparación con los programas que no cumplen con estas premisas. 
La última fase incluye la evaluación final del programa, elemento esencial para promover una intervención eficaz y que busca mejorar la calidad del programa para futuras intervenciones. Es importante que para esta evaluación se usen, en la medida de lo posible, pruebas contrastadas y validadas mediante otros estudios.

\section{Programa SEA}

En base al estudio de las necesidades socioemocionales y al estudio de la literatura del área, se diseñó el programa de desarrollo de competencia Socioemocionales SEA (CelmaPastor y Rodríguez-Ledo, 2017). Este modelo se fundamenta en modelos de habilidad (Bisquerra, 2003) y mixtos (Bar-On, 2006) de modo que aglutine de un modo práctico y simple los tres grupos de competencias socioemocionales en la base de la ISE. Con este objetivo práctico se busca que los tutores sean conscientes que competencia concreta están potenciando en cada momento y así hacer del programa uno eficaz y eficiente. Con respecto al programa en sí, se diseña primero en su forma piloto con 10 sesiones de 55' de trabajo que se aplican en la hora de tutoría de un IES público de la ciudad de Zaragoza, tras lo cual se completa y mejora con la selección de nuevas sesiones y actividades hasta que obtiene su versión final de 18 sesiones de 55' de trabajo. El programa SEA cumple con las fases de formación previa y evaluación de resultados posterior, así como con S.A.F.E. basándose en el modelo de inteligencia socioemocional SEA (Celma-Pastor y Rodríguez-Ledo, 2017) que entiende la ISE como la capacidad de las personas para percibir y comprender los estados emocionales propios y de los demás, así como para regularlos y expresarlos de un modo adaptativo socialmente y que define esta como formada por tres grupos de habilidades a potenciarse en el aula. El primero, atención y comprensión emocional, se entiende como la disposición a atender, comprender y aceptar las emociones y estados de ánimo propios y ajenos. El segundo, regulación y reparación emocional, se define como la capacidad de regular internamente nuestras emociones ante las distintas situaciones. La capacidad para reparar y mantener un estado emocional concreto o eliminar otro que nos puede resultar negativo es una habilidad clave de esta dimensión. Por último, el tercero de los grupos, expresión social adaptativa de las emociones, explica la capacidad para acomodar nuestra conducta social de acuerdo al contexto en el que nos encontremos, para así adecuarnos a él y hacer uso de todas nuestras habilidades interpersonales o sociales. 


\section{Objetivos e hipótesis}

El objetivo general del programa es, por tanto, el de potenciar el desarrollo de estas competencias socioemocionales y con ellas la ISE general de los participantes del mismo. Nuestra hipótesis de trabajo en el estudio que aquí se presenta fue, por lo tanto, la de comprobar si la aplicación del programa SEA durante un curso escolar mejora las competencias ISE apenas definidas en los participantes del mismo.

\section{Método}

\section{Participantes}

La muestra de este estudio estuvo formada por 156 adolescentes de 11 a 14 años, distribuidos en las seis aulas de $1^{\circ}$ de ESO de un instituto de educación secundaria; el centro se encuentra ubicado en un barrio obrero de la ciudad de Zaragoza. Cuatro de estas clases fueron asignadas aleatoriamente a la condición experimental (108 participantes) y las otras dos a la de control (48 estudiantes). En cuanto al género, 86 son chicos $(55,1 \%)$ y 70 son chicas (44,9\%). De los 108 participantes experimentales, 58 (53,7\%) son varones y $50(46,3 \%)$ son mujeres, mientras que, de los 48 participantes de control, 28 (58,3\%) son varones y 20 $(41,7 \%)$ son mujeres. Además, la distribución de los sexos en las dos condiciones es equivalente de manera estadísticamente significativa $\left(X^{2}=0.592\right.$ y $\left.\mathrm{p}>0.05\right)$. El nivel socioeconómico y cultural de la muestra es medio. Los padres, madres y tutores legales de los participantes fueron informados, por escrito y en una reunión, de las características de la investigación; todos ellos dieron de manera explícita el consentimiento para realizar este estudio. Se garantizó y respetó el anonimato de las respuestas y puntuaciones de todos los participantes.

\section{Instrumentos}

Competencias socioemocionales: Cuestionario de Desarrollo Emocional para Secundaria, CDE-SEC (Álvarez et al., 2001). Este cuestionario de autoinforme consta de 35 ítems a los que se responde según el grado de acuerdo o desacuerdo siendo 0 completamente en desacuerdo, y 10 completamente de acuerdo. Se evalúan las dimensiones socioemocionales pro-puestas en los trabajos del Grupo de investigación GROP y su modelo de competencias socioemocionales mixto (Bisquerra y Pérez, 2007): 1) Conciencia emocional: Capacidad para tomar conciencia de las propias emociones, incluyendo la habilidad para captar el clima emocional de un contexto determinado; 2) Regulación emocional: Capacidad para utilizar las 
emociones de forma adecuada y de tener buenas estrategias de "afrontamiento", capacidad para autogenerarse emociones positivas; 3) Autonomía emocional: características relacionadas con la autogestión emocional, tales como autoestima, actitud positiva en la vida, responsabilidad, capacidad para analizar crítica-mente las normas sociales, capacidad para buscar ayuda y recursos, y como la autoeficacia personal; 4) Competencias sociales: capacidad para mantener buenas relaciones con otras personas y dominar las habilidades sociales básicas, comunicación efectiva, respeto, actitudes pro-sociales, asertividad, etc.; 5) Competencias para la vida y el bienestar: Capacidad para adoptar comportamientos apropiados y responsables para la solución de problemas personales, familiares, profesionales y sociales, orientados hacia la mejora del bienestar de vida personal y social. Además, se puede obtener también una puntuación total de competencia socioemocional, producto de la media de las demás variables. La fiabilidad de la prueba varía entre $\alpha=0.79$ y $\alpha=0.82$, para cada una de las dimensiones (Pérez-Escoda, 2016).

Competencias socioemocionales: Cuestionario de inteligencia emocional, versión para jóvenes EQi-YV (Ferrándiz, Hernández, Bermejo, Ferrando y Sáinz, 2012). El EQi-YV es un cuestionario de autoinforme diseñado para medir la IE de niños y adolescentes de 7 a 18 años basada en la prueba original EQ (Bar-On, 2006) y adaptada a muestras españolas. Se trata de una escala tipo Likert cuyos 60 ítems dan lugar a cinco grandes dimensiones del modelo mixto de Bar-On (2006) y que permiten el estudio de las siguientes características emocionales y sociales de los sujetos: 1) Competencia intrapersonal: habilidad para comprender las propias emociones y su comunicación a los otros; 2) Competencia interpersonal: habilidad para entender y apreciar las emociones de los otros; 3) Manejo de las emociones: habilidad para dirigir y controlar las propias emociones; 4) Adaptabilidad: flexibilidad y eficacia para adaptarse al ambiente social y resolver conflictos; 5) Estado de ánimo general: habilidad para tener una actitud positiva ante la vida. Se trata de un inventario amplio que proporciona información acerca de las competencias emocionales y sociales y que permite trazar un perfil social y afectivo. La validación en muestra española obtiene una fiabilidad que oscila entre $\alpha$ $=0.63$ (competencia intrapersonal) y $\alpha=0.80$ (estado de ánimo) para las cinco dimensiones (Ferrándiz et al., 2012). Esta prueba se incorporó sólo en el postest debido a que cuando se realizó la medida pretest no había sido aún validada en muestras españolas.

Ajuste social y habilidades sociales: Batería de socialización, BAS3 (Silva y Martorell, 2001). La batería de socialización BAS3 consta de 75 ítems en esta versión de auto$-690-$ 
aplicación. Evalúa cinco dimensiones que nos permiten conocer el perfil de la conducta social del evaluado y su ajuste social, a saber: 1) Consideración con los demás: detecta sensibilidad social o preocupación por los demás, en particular por aquellos que tienen problemas y son rechazados o postergados; 2) Auto-control en las relaciones sociales: recoge una dimensión claramente bipolar que representa, en su polo positivo, acatamiento de reglas y normas sociales que facilitan convivencia en el mutuo respe-to, y en el polo negativo, conductas agresivas, impositivas, de terquedad e indisciplina; 3) Retrai-miento social: detecta apartamiento tanto pasivo como activo de los demás, hasta llegar, en el ex-terno, a un claro aislamiento; 4) Ansiedad Social/Timidez: se detectan distintas manifestaciones de ansiedad unidas a reacciones de timidez en las relaciones sociales; 5) Liderazgo: se detecta as-cendencia, popularidad, iniciativa, confianza en sí mismo y espíritu de servicio. Se trata de una prueba altamente fiable que presenta una consistencia interna media de $\alpha=0.75$ y una estabilidad test-retest de $\alpha=$ 0.57 medida con el alpha de Cronbach (Silva y Martorell, 2001).

Empatía: Escala de Empatía para Niños y Adolescentes (IECA; Index of Empaty for Children and Adolescents) (Bryant, 1982; Mestre, Pérez-Delgado, Frías y Samper, 1999). Esta escala consta de 22 ítems de respuesta sí o no en función del acuerdo o no del evaluado ante 22 situaciones donde la empatía personal se pone a prueba. Se trata de una adaptación para la población infantil y adolescente (a partir de 11 años) de la escala para adultos de Mehrabian y Epstein (1972) llevada a cabo por Bryant (1982). En concreto, se trata de una escala que aporta un índice general de empatía, y cuya consistencia interna de la escala original es de 0.67 (Bryant, 1982). En el caso de la muestra española, la escala presenta una fiabilidad testretest entre $\alpha=0.75$ y $\alpha=0.77$ en función de la muestra utilizada por la investigación española de la que se ha obtenido (Mestre et al., 1999). En esta investigación el alpha de Cronbach obtenido fue de $\alpha=0.77$.

\section{Procedimiento}

Se utilizó un diseño pre-test/post-test cuasi-experimental con grupo de control para evaluar la eficacia del programa de intervención. Los aplicadores del programa en los 4 grupos-clase que componen el grupo experimental fueron los propios tutores. Los instrumentos utilizados fueron administrados por los tutores de los grupos-clase en la condición experimental y en el control. La implementación del programa SEA (Celma Pastor y Rodríguez Ledo, 2017) supuso 9 meses y fue precedida de la formación de los tutores aplicadores de la condición experimental. Esta formación versó sobre la importancia de potenciar la ISE en el aula, el 
modelo teórico de base del programa y técnicas concretas de aplicación de las diferentes sesiones en base a las actividades concretas del mismo. Además, se llevó a cabo un seguimiento semanal mediante reuniones formales e informales con los mismos para comprobar la correcta aplicación del programa y ofrecer ayuda y consejos en base a las actividades concretas.

Para evaluar la eficacia del programa (variable independiente) se estudiaron tres variables dependientes concretas: la mejora en competencias socioemocionales, la mejora en empatía y la mejora en ajuste social y habilidades sociales. La primera de las variables dependientes se estudió, además de en las medidas pre-test y post-test al acabar la intervención, 3 meses después con el objetivo de observar si tales mejoras se mantenían en el tiempo.

\section{Análisis de datos}

Debido a la distribución normal de los errores y de la relación lineal de las variables dependientes e independientes, los datos de competencia emocional (pre-postest y seguimiento), la empatía y la medida de ajuste social se sometieron a un análisis de modelo lineal general. Para cada medida se aplicó el modelo univariante ANCOVA, donde la variable dependiente es la medida post-test, la covariable la medida pre-test y el factor fijo la condición experimental. En el caso de la medida de competencia socioemocional de seguimiento, al no disponer de medida pre intervención de esta variable se tomó como covariable la medida total de competencia emocional de la prueba CDE-SEC, ya que se puede considerar como el mejor indicador de competencia emocional en la medida pre-test. Los estadísticos utilizados mediante ANCOVA para medir el efecto fueron la traza de Pillai $(F)$ y $\eta^{2}$ p, que mide el tamaño del efecto, siendo $\eta^{2} p<0,06$ pequeño, $\eta^{2} p>0,06$ y $<0,14$ medio y $\eta^{2} p>0,14$ grande.

\section{Resultados}

\section{Descriptivos}

La tabla 1 muestra los estadísticos descriptivos de cada variable medida en este estudio en su medida pre-test y post-test para cada una de las condiciones experimentales. 
Tabla 1. Estadísticos descriptivos

\begin{tabular}{|c|c|c|c|c|c|}
\hline & & \multicolumn{2}{|c|}{ Pre-test } & \multicolumn{2}{|c|}{ Post-test } \\
\hline \multirow{23}{*}{ 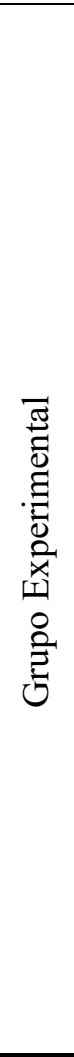 } & & Media & DT & Media & DT \\
\hline & Competencias socioemocionales (CDE-SEC) & & & & \\
\hline & Conciencia emocional & 7,95 & 1,188 & 7,87 & 1,381 \\
\hline & Regulación emocional & 5,12 & 1,533 & 5,31 & 1,479 \\
\hline & Autonomía emocional & 6,09 & 1,543 & 5,96 & 1,509 \\
\hline & Competencia social & 6,14 & 1,605 & 6,15 & 1,601 \\
\hline & Competencias de vida y bienestar & 7,39 & 1,510 & 7,04 & 1,455 \\
\hline & Competencia emocional total & 6,46 & 1,078 & 6,39 & 1,034 \\
\hline & Competencias socioemocionales (EQi-YV) & & & & \\
\hline & Intrapersonal & --- & --- & 15,29 & 3,128 \\
\hline & Interpersonal & --- & --- & 39,75 & 4,667 \\
\hline & Manejo del estrés & --- & --- & 32,71 & 5,765 \\
\hline & Adaptabilidad & --- & --- & 29,31 & 4,935 \\
\hline & Estado de ánimo general & --- & --- & 46,86 & 5,747 \\
\hline & Inteligencia socioemocional total & --- & --- & 163,92 & 14,970 \\
\hline & Empatía (IECA) & & & & \\
\hline & Empatía general & 15,93 & 3,656 & 16,29 & 3,929 \\
\hline & Ajuste social y habilidades sociales (BAS3) & & & & \\
\hline & Consideración de los demás & 11,89 & 2,489 & 11,95 & 2,404 \\
\hline & Autocontrol social & 10,26 & 3,432 & 9,70 & 3,492 \\
\hline & Retraimiento social & 1,51 & 2,053 & 1,38 & 1,984 \\
\hline & Ansiedad social-timidez & 3,77 & 2,826 & 3,92 & 2,882 \\
\hline & Liderazgo & 5,84 & 2,260 & 6,83 & 2,523 \\
\hline \multirow{22}{*}{ 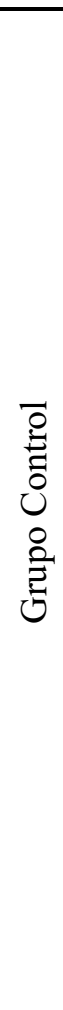 } & Competencias socioemocionales (CDE-SEC) & & & & \\
\hline & Conciencia emocional & 7,83 & 1,391 & 7,55 & 1,287 \\
\hline & Regulación emocional & 5,13 & 1,402 & 5,07 & 1,453 \\
\hline & Autonomía emocional & 6,12 & 1,577 & 5,84 & 1,621 \\
\hline & Competencia social & 5,99 & 1,459 & 6,25 & 1,499 \\
\hline & Competencias de vida y bienestar & 7,29 & 1,414 & 6,80 & 1,609 \\
\hline & Competencia emocional total & 6,40 & 0,907 & 6,23 & 1,063 \\
\hline & Competencias socioemocionales (EQi-YV) & & & & \\
\hline & Intrapersonal & --- & --- & 15,03 & 3,219 \\
\hline & Interpersonal & --- & --- & 36,62 & 4,487 \\
\hline & Manejo del estrés & --- & --- & 30,78 & 5,350 \\
\hline & Adaptabilidad & --- & --- & 26,19 & 4,742 \\
\hline & Estado de ánimo general & --- & --- & 45,97 & 5,881 \\
\hline & Inteligencia socioemocional total & --- & --- & 154,59 & 16,597 \\
\hline & Empatía & & & & \\
\hline & Empatía general & 15,50 & 3,307 & 14,87 & 3,955 \\
\hline & Ajuste social y habilidades sociales (BAS3) & & & & \\
\hline & Consideración de los demás & 11,62 & 2,385 & 10,85 & 2,646 \\
\hline & Autocontrol social & 9,83 & 2,985 & 8,89 & 3,571 \\
\hline & Retraimiento social & 1,10 & 1,372 & 1,89 & 2,672 \\
\hline & Ansiedad social-timidez & 3,17 & 2,853 & 3,38 & 3,254 \\
\hline & Liderazgo & 6,08 & 2,305 & 6,32 & 2,433 \\
\hline
\end{tabular}


Resultados de mejora del programa

Los resultados obtenidos tras aplicar el modelo lineal general univariante ANCOVA se muestran en la tabla 2. En ella se pueden observar los cambios pre/post-test introducidos, presumiblemente, por el programa, medidos mediante la traza de Pillai (F), siempre con 1 grado de libertad, el tamaño del efecto $\left(\eta^{2} \mathrm{p}\right)$ y la significación estadística de los mismos $(\mathrm{p})$.

Tabla 2. Resultados generales de modelo lineal general

\begin{tabular}{lccc}
\hline & $\mathrm{F}$ & $\eta^{2} \mathrm{p}$ & $\mathrm{p}$ \\
\hline Competencias socioemocionales (CDE-SEC) & & & \\
Conciencia emocional & 0,985 & 0,007 & 0,323 \\
Regulación emocional & 1,563 & 0,011 & 0,213 \\
Autonomía emocional & 0,334 & 0,002 & 0,564 \\
Competencia social & 0,371 & 0,003 & 0,544 \\
Competencias de vida y bienestar & 0,369 & 0,003 & 0,545 \\
Competencia emocional total & 0,682 & 0,005 & 0,410 \\
\hline Competencias socioemocionales (EQi-YV) & & & \\
Intrapersonal & 0,597 & 0,005 & 0,441 \\
Interpersonal & 11,040 & 0,088 & 0,001 \\
Manejo del estrés & 2,934 & 0,025 & 0,089 \\
Adaptabilidad & 10,022 & 0,080 & 0,002 \\
Estado de ánimo general & 0,281 & 0,002 & 0,597 \\
Inteligencia socioemocional total & 10,244 & 0,082 & 0,002 \\
\hline Empatía (IECA) & & & \\
Empatía general & 4,070 & 0,027 & 0,045 \\
\hline Ajuste social y habilidades sociales (BAS3) & & & \\
Consideración de los demás & 7,158 & 0,046 & 0,008 \\
Autocontrol social & 1,537 & 0,010 & 0,217 \\
Retraimiento social & 4,371 & 0,028 & 0,038 \\
Ansiedad social-timidez & 0,236 & 0,002 & 0,628 \\
Liderazgo & 2,726 & 0,018 & 0,101 \\
\hline \multicolumn{1}{c}{ Nota. N para el grupo experimental =108 y N para el grupo control = 48 }
\end{tabular}

Nota. $\mathrm{N}$ para el grupo experimental $=108$ y N para el grupo control $=48$

Como se puede observar los resultados no ofrecen diferencias significativas en ninguna de las variables de competencia emocional medidas mediante la prueba CDE-SEC. De hecho, los participantes experimentales informan de un cierto decremento de competencias socioemocionales en la medida post-test que puede relacionarse con el decremento de habilidades en las primeras fases de la adolescencia (Mariscal et al., 2009; Pasch et al., 2008). En este sentido, los resultados obtenidos con el grupo control no solo informan también de este descenso, sino que es aún mayor que en los participantes experimentales, pudiendo deberse a un efecto protector del programa de intervención consistente con la literatura (Extremera y Fernández-Berrocal, 2003; Greenberg et al., 2003; Humphrey et al., 2007). Más allá de este resultado que, sin embargo, no obtiene puntuaciones significativas, un estudio más exhaustivo 
del efecto de la intervención en la ISE de los participantes mediante la la prueba de competencias socioemocionales EQi-YV sí informa de que existen diferentes significativas entre la mejora en los participantes del grupo experimental por encima de los del grupo control. En concreto se observan mejora en las variables interpersonal $\left[F(1,118)=11,040, \eta^{2} p=0,088, p\right.$ $<0,01]$, adaptabilidad $\left[F(1,118)=10,022, \eta^{2} \mathrm{p}=0,080, p<0,01\right]$ y en la dimensión total de competencia socioemocional $\left[F(1,118)=10,244, \eta^{2} p=0,082, p<0,01\right]$, siendo los tamaños del efecto medios en todas ellas. También en la variable empatía, medida con la prueba IECA, se observa como existe una diferencia significativa entre experimentales y controles en la medida de empatía general medida $\left[F(1,151)=4,070, \eta^{2} p=0,045, p<0,05\right]$, observándose un tamaño del efecto pequeño. Por último, en relación a las variables de ajuste social, medidas con el BAS3, se observan diferencias significativas en dos de las dimensiones: consideración de los demás $\left[F(1,152)=7,158, \eta^{2} p=0,046, p<0,01\right]$ y retraimiento social $[F(1,152)=$ $\left.4,371, \eta^{2} p=0,028, p<0,05\right]$, ambas con tamaños del efecto pequeños.

\section{Discusión y Conclusiones}

El objetivo del estudio presentado en este artículo era el de evaluar el impacto de la primera implementación del programa de desarrollo de competencias socioemocionales SEA con alumnado joven adolescente. La aplicación de sus 18 sesiones de 55' de duración con 106 participantes de $1^{\circ}$ de E.S.O. por parte los propios profesores-tutores de los grupos clase se diseñó en base al modelo teórico en la base del programa, el modelo SEA (Celma-Pastor y Rodríguez-Ledo, 2017). Este modelo de desarrollo de competencias socioemocionales entiende la ISE como la capacidad de las personas para percibir y comprender los estados emocionales propios y de los demás, así como para regularlos y expresarlos de un modo adaptativo socialmente.

El impacto en la ISE de los participantes se evaluó mediante dos pruebas de competencias socioemocionales, una pretest-postest y otro en formato de seguimiento, ambas basadas en modelos de la ISE mixtos y cercanos al nuestro propio. Sumado a estas se usan otras dos pruebas también en modo pretest-postest que analizan la mejora en empatía y el desarrollo de habilidades sociales y ajuste social. Los resultados obtenidos con estas pruebas nos informaron de que, en efecto, la aplicación del programa SEA resulta en la mejora de los participantes del mismo, por encima de aquellos controles que no trabajan con él, en competencias ISE relacionadas con los aspectos más sociales de la ISE. Así, estos alumnos mejoraron en la 
capacidad para expresar sus emociones de un modo más adaptativo, desarrollando más competencias interpersonales, así como de adaptabilidad al medio. También mejoraron en la comprensión de estados emocionales de los demás, como se observa en las mejoras encontradas en empatía y en consideración de los demás. Además, los participantes experimentales del estudio informaron sentirse con mayores competencias socioemocionales o de ISE totales. Por otro lado, los resultados parecen indicar que el programa SEA puede prevenir el descenso de competencias socioemocionales típicas de la adolescencia (Mariscal et al., 2009; Pasch et al., 2008), lo que es consistente con la literatura en el campo (Extremera y Fernández-Berrocal, 2003; Greenberg et al., 2003; Humphrey et al., 2007). Por último, el programa SEA participa en la creación de alumnos más ajustados a su centro educativo y aula en tanto que inician conductas sociales con mayor facilidad, como se desprende de las mejoras en las puntuaciones de retraimiento social.

Los resultados obtenidos suponen un apoyo al programa en sí, así como al hecho de que sea implementado por los propios tutores de las aulas donde se desarrolle. Que las aplicaciones de programas de desarrollo competencias socioemocionales llevadas a cabo por profesores pueden ser efectivas para desarrollar la ISE de sus alumnos está contrastado en otros estudios (Durlak et al., 2011; Zins, Weissberg, Wang y Walberg, 2004). Por otro lado, como indican Nelis, Quoidbach, Mikolajczak y Hansenne (2009) la mayoría de los programas impelementados no están fundamentados en bases teóricas concretas, carecen de objetivos bien delimitados y no utilizan grupos control para evaluar las mejoras introducidas por el programa. Además, muchos de estos programas no cumplen con un diseño S.A.F.E. (Durlak et al., 2011) que incremente la posibilidad de que el programa sea no solo eficaz sino eficiente. El presente estudio si presenta tales características de diseño, implementación y evaluación, que sin embargo no le exime de cierto campo de mejora.

Una de estas mejoras se relaciona con los tamaños del efecto que, si bien descubren mejoras interesantes en una sola aplicación relativamente corta del programa, son siempre medios o pequeños, existiendo un margen de mejora. Otra de las limitaciones se relaciona con las pruebas de auto-informe utilizadas, que ofrecen una información no exenta de subjetividad por parte de los participantes de las mismas. Estas, sin embargo, han sido inevitables, en tanto que no existen medidas objetivas para alumnado de estas edades del mismo modo que la aplicación de este tipo, en caso de que existieran, supondría un incremento de tiempo mucho mayor que podría entrar en conflicto con el tiempo de formación necesario para jóvenes de estas $-696-$ 
edades. Sin embargo, el diseño de tales pruebas objetivas mejoraría la fiabilidad de los resultados y así lo recomendamos, sobre todo en pruebas lo más cortas posibles. Por otro lado, este efecto protector encontrado mediante la prueba CDE-SEC ha de contrastarse de nuevo, a poder ser mediante el uso de esta y otras pruebas de medida de competencias socioemocionales análogas. Por último, la muestra de 156 participantes (108 experimentales) nos hace plantearnos la validez externa de esto resultados en tanto que potencialmente extrapolables a jóvenes de tales edades del territorio español. Si bien los participantes pertenecen a grupos regulares, lo que incrementa su representatividad general, sugerimos a futuros investigadores que incrementen el número de participantes de este programa, así como evalúen el posible impacto en otros grupos con características propias como alumnado con TDAH o con necesidades educativas diversas.

Más allá de las posibles futuras mejoras concretas en términos de investigación, está claro que el objetivo principal de todos aquellos expertos en educación y psicología del desarrollo debe estar en mejorar nuestro sistema educativo en tanto que primordial para desarrollar personas con un buen set competencias para la vida, entre ellas las competencias socioemocionales. Si sabemos que los jóvenes con más ISE serán previsiblemente adultos con mejor ajuste profesional, informantes de poseer un mayor bienestar (Zeidner y Matthews, 2016) y, que la formación en ISE puede predecir el éxito académico tanto como la personalidad y no mucho peor que las habilidades cognitivas (Barchard, 2003), nuestra obligación es formar para el desarrollo personal y profesional, y así potenciar su futuro éxito en ambos campos. En este proceso el programa aquí presentado supone una propuesta de éxito para desarrollar la ISE de aquellos que lo trabajan, convirtiéndose así en una propuesta de intervención a sumarse a muchas otras que buscan el tan ansiado fin que todo sistema educativo anhela: desarrollar personas competentes en todos los ámbitos de su vida.

\section{Referencias}

Álvarez, M. (coord), Bisquerra, R., Filella, G., Fita, E., Martínez, F. y Pérez-Escoda, N. (2001): Diseño y evaluación de programas de educación emocional. Barcelona: Praxis. ISSN: 1575-023X1695-5714.

Akerjordet, K. y Severinsson, E. (2007). Emotional intelligence: A review of the literature with specific focus on empirical and epistemological perspectives. Journal of Clinical Nursing, 16(8), 1405-1416. DOI: 10.1111/j.1365-2702.2006.01749.x. 
Barchard, K. A. (2003). Does emotional intelligence assist in the prediction of academic success? Educational and Psychological Measurement, 63(5), 840-858. Doi: $10.1177 / 0013164403251333$.

Bar-On, R. (2006). The Bar-On model of emotional-social intelligence (ESI). Psicothema,18(Suppl), 13-25. ISSN 1886-144X (Electronic); 0214-9915 (Print).

Bisquerra, R. (2003). Educación emocional y competencias básicas para la vida. Revista de Investigación Educativa, 21(1), 7-43.

Bisquerra, R. y Pérez, N. (2007). Las competencias emocionales. Educación XXI, 10, 61-82. Doi: $10.5944 /$ educxx1.

Brackett, MA, Patti, J., Stern, R., Rivers, S.E., Elbertson, Chisholm, C. y Salovey, P. (2009). A sustainable, skill-based approach to building emotionally literate schools. En M. Hughes, H. L. Thompson y J. B. Terrell (Eds.), Handbook for Developing Emotional and Social Intelligence: Best Practices, Case Studies, and Strategies (pp.329-357). New Jersey: John Wiley \& Sons. ISBN: 978-0-470-19088-3.

Bryant, B. K. (1982). An Index of Empathy for Children and Adolescents. Child Development , 53(2), 413-425. Doi: 10.2307/1128984.

CASEL (Collaborative for Academic, Social, and Emotional Learning). (2015). CASEL Guide: Effective Social and Emotional Learning Programs, Middle and High School Edition. Recuperado de http://secondaryguide.casel.org/casel-secondary-guide.pdf.

Celma-Pastor, L. y Rodríguez-Ledo, C. (2017). Educación Socioemocional para jóvenes en el Aula: Programa SEA. Madrid: TEA Ediciones. ISBN: 978-84-16231-50-8.

Ciarrochi, J., Chan, A. C. y Bajgar, J. (2001). Measuring emotional intelligence in adolescents. Personality and Individual Differences, 31(7), 1105-1119. Doi: 10.1016/S01918869(00)00207-5.

Durlak, J. A., Weissberg, R. P., y Pachan, M. (2010). A meta-analysis of after-school programs that seek to promote personal and social skills in children and adolescents. American journal of community psychology, 45(3-4), 294-309. Doi: 10.1007/s10464010-9300-6.

Durlak, J. A., Weissberg, R. P., Dymnicki, A. B., Taylor, R. D. y Schellinger, K. B. (2011). The Impact of Enhancing Students' Social and Emotional Learning: A Meta-Analysis of School Based Universal Interventions. Child Development, 82(1), 405-432. Doi: 10.1111/j.1467-8624.2010.01564.x. 
Durlak, J. A. y Wells, A. M. (1997). Primary prevention mental health programs for children and adolescents: A meta-analytic review. American Journal of Community Psychology, 25, 115-152. Doi: 10.1023/A:1024654026646.

Ekman, P. y Davidson, R. (1994). The nature of emotion: fundamental questions. Nueva York: Oxford University Press. ISBN: 9780195089448.

Extremera, N. y Fernández-Berrocal, P. (2003). La inteligencia emocional en el aula como factor protector de conductas problema: violencia, impulsividad y desajuste emocional. Actas I Congreso Hispanoamericano de Educación y Cultura de Paz, 599-605. Editorial Universidad de Granada: Granada.

Fernández-Berrocal, P. y Ruiz-Aranda, D. (2008). La inteligencia emocional en la educación. Revista de Investigación Psicoeducativa, 6.,193-204. ISSN. 1696-2095.

Ferrándiz, C., Hernández, D., Bermejo,R., Ferrando, M. y Sáinz, M. (2012). Social and Emotional Intelligence in Childhood and Adolescence: Spanish Validation of a Measurement Instrument. Revista de Psicodidáctica, 17(2), 309-338. Doi:10.1387/Rev.Psicodidact.2814

Feshbach, N.D. (1978) Studies of empathic behavior in children. En B.A. Maher (Ed.), Progress in experimental personality research (pp.1-47). Nueva York: Academic Press. ISBN: 978-0-12-541413-5.

Gomez-Baya, D., Mendoza, R., Paíno, S. y Gaspar de Matos, M. (2017). Perceived emotional intelligence as a predictor of depressive symptoms during mid-adolescence: A twoyear longitudinal study on gender differences. Personality and Individual Differences, 104. 303-312. Doi: 10.1016/j.paid.2016.08.022.

Greenberg, M. T., Weissberg, R. P., O'Brien, M. U., Zins, J. E., Fredericks, L., Resnik, H. y Elias, M. J. (2003). Enhancing school-based prevention and youth development through coordinated social, emotional, and academic learning. American psychologist, 58(6-7), 466-474. Doi: 10.1037/0003-066X.58.6-7.466.

Humphrey, N., Curran, A., Morris, E., Farrel, P. y Woods, K. (2007). Emotional Intelligence and education: A crtitical review. Educational Psychology, 27(2), 235-254. Doi: $10.1080 / 01443410601066735$.

Lopes, P. N., Salovey, P. y Straus, R. (2003). Emotional intelligence, personality, and the perceived quality of social relationships. Personality and Individual Differences, 35(3), 641-658. Doi: 10.1016/S0191-8869(02)00242-8.

Mariscal, S., Giménez-Dasí, M., Carriedo, N. y Corral, A. (2009). El desarrollo psicológico a lo largo de la vida. UNED: Mc Graw Hill. ISBN: 9788448171285. 
Martín Jorge, M., Harillo García, D. y Mora Mérida, J. (2008). Relaciones entre inteligencia emocional y ajuste psicológico en una muestra de alumnos de enseñanza secundaria. Ansiedad y Estrés, 14(1), 31-44. ISSN: 1134-7937.

Mayer, J., Salovey, P. y Caruso, D. (2000). Emotional intelligence as zeitgeist, as personality, and as a mental ability. En R. Bar-On, The handbook of emotional intelligence: Theory, development, assessment, and application at home, school, and in the workplace. (pp. 92-117). San Francisco: Jossey-Bass. ISBN: 978-0470907436.

Mehrabian, A. y Epstein, N. A. (1972). A measure of emotional empathy. Journal of Personality, 40(4), 525-543. Doi: 10.1111/j.1467-6494.1972.tb00078.x.

Mestre, V., Pérez-Delgado, E., Frías, D. y Samper, P. (1999). Instrumentos de evaluación de la empatía. En E. Pérez-Delgado y V. Mestre, Psicología moral y crecimiento personal (pp.181-190). Barcelona: Ariel. ISBN: 84-344-0883-X

Nelis, D., Quoidbach, J., Mikolajczak, M., \& Hansenne, M. (2009). Increasing emotional intelligence:(How) is it possible? Personality and Individual Differences, 47(1), 36-41. Doi: 10.1016/j.paid.2009.01.046

Pasch, K. E., Nelson, M. C., Lytle, L. A., Moe, S. G. y Perry, C. L. (2008). Adoption of risk related factors through early adolescence: Associations with weight status and implications for causal mechanisms. Journal of Adolescent Health, 43, 387-393. Doi: 10.1016/j.jadohealth.2008.02.009.

Pérez-Escoda, N. (2016). Cuestionarios del GROP para la evaluación de la competencia emocional (CDE). En Soler, J. L.; Aparicio, L.; Díaz, J.; Escolano, E.; Rodríguez, M.A. Inteligencia emocional y bienestar II. Reflexiones, experiencias profesionales e investigaciones (pp. 690-705). Zaragoza: Ediciones Universidad San Jorge. ISBN: 978-84$608-4847-9$

Petrides, K. y Furnham, A. (2000). On the dimensional structure of emotional intelligence. Personality and Individual Differences, 29, 313-320. Doi: 10.1016/S01918869(99)00195-6.

Resurrección, D., Salguero, J. y Ruiz-Aranda, D. (2014). Emotional intelligence and psychological maladjustment in adolescence: A systematic review. Journal of Adolescence, 37. 461-472. Doi: 10.1016/j.adolescence.2014.03.012.

Rodríguez-Ledo, C. y Ruíz-Aranda, D. (2017). Inteligencia y educación socioemocional. En A. Cortés Pascual y A. Rodríguez Martínez (Coords.), Una mirada psicosocioeducativa. Innovación colectiva para el desarrollo personal y profesión. Anaya: En prensa. 
Sanz de Acebo, M. L., Ugarte, M. D., Cardelle-Elawar, M., Iriarte, M. D. y Sanz de Acebo, M. T. (2003). Enhancement of self-regulation, assertiveness, and empathy. Learning and Instruction, 13(4), 423-439. Doi: 10.1016/S0959-4752(02)00026-9.

Silva, F. y Martorell, C. (2001). BAS-3: Batería de Socialización (Autoevaluación). Madrid: TEA. ISBN: 8471742640978847174264384717463959788471746399.

Schutte, N. S., Malouff, J., Bobik, C., Coston, T., Greeson, C., Jedlicka, C. et al. (2001). Emotional intelligence and interpersonal relations. Journal of Social Psychology, 141(4), 523-536. Doi: 10.1080/00224540109600569.

Wilson, S. J. y Lipsey, M. W. (2007). School-based interventions for aggressive and disruptive behavior: Update of a meta-analysis. American Journal of Preventive Medicine, 33(Suppl.2S), 130-143. Doi: 10.1016/j.amepre.2007.04.011.

Zins, J. E., Weissberg, R. P., Wang, M. C., y Walberg, H. J. (Eds.). (2004). Building academic success on social and emotional learning: What does the research say? Nueva York: Teachers College Press. ISBN: 978-0807744390.

Zeidner, M. y Matthews, G. (2016). Ability emotional intelligence and mental health: Social support as a mediator. Personality and Individual Differences, 99. 196-199. Doi: 10.1016/j.paid.2016.05.008 